\title{
'High-risk' patients with chronic obstructive pulmonary disease (COPD) have a poor prognosis and need to be able to access palliative care services
}

\author{
Authors: Qamar Abbas Syed, ${ }^{1}$ Michael Apps, ${ }^{2}$ Dipak Mukherjee, ${ }^{1}$ Munachi Nkere, ${ }^{1}$ Sylvia Aneke ${ }^{1}$ and $]$ Minter ${ }^{2}$
}

\begin{abstract}
Aims
To assess the care need of COPD patients who have high risk of recurrent admissions and death.

\section{Methods}

We have collected data prospectively on a group of patients with COPD who have had three or more admissions in the previous year or who have been referred to the COPD multidisciplinary team (MDT) or both. We have examined these patients to identify the severity and complexity of their disease, potential interventions and their clinical course, including admissions and prognosis after the date of identification.
\end{abstract}

\section{Results}

We have collected data on 76 patients with COPD, 33 men and 43 women, age range $41-90$ years. We have spirometry on $66 \%$ of patients (50/76), showing 12\% GOLD stage 1-2, 14\% GOLD stage 3 and $74 \%$ GOLD stage 4 .

On average, each patient had 3.9 comorbid conditions. They had been admitted to hospital three or more times for those identified from multiple admissions, and 3.5 times $(0-13)$ in those discussed at MDT. They had complex disease, with $70 \%$ on nebulised therapy, $49 \%$ on oxygen, $28 \%$ on non-invasive ventilation (NIV) or CPAP, and 2/76 on colomycin therapy for treatment for persistent Pseudomonas infection. Despite attempts at smoking cessation on multiple occasions, $37 \%$ were still smoking.

In the period after identification, 36/76 (47\%) died between the time of identification for the study and 1 October 2016. $12 \%$ of these died within 1 month of identification, $42 \%$ within 3 months, $70 \%$ within 6 months and $97 \%$ within 12 months. Only $50 \%$ of those who died and $37 \%$ of the whole group had contact with hospice or other palliative care services.

Interventions were carried out where it was identified that: There was a BMI of $<17$, when they were referred for nutritional support; home NIV was indicated because of chronic CO2 retention; social are packages and admission to a care home were necessary; the patient was smoking when smoking cessation support was offered.

$33 \%$ of patients were referred for pulmonary rehabilitation, although a fifth of these declined to do it.

\section{Conclusions}

There are many patients with COPD who have a 'high risk' of deterioration and death. The prognosis for this group is poor, with almost half dying within a year, yet only $37 \%$ had accessed palliative care services $50 \%$ in those who died during followup). More palliative care input is needed for patients with severe COPD, especially those discussed at MDT or with multiple admissions to hospital.

\section{Conflict of interest statement}

None.

Authors: ${ }^{1}$ Basildon and Thurrock University Hospital, Basildon, UK; ${ }^{2}$ NELFT NHS Foundation Trust, Basildon, UK 